\title{
Editorial: Dossier microbiología oral y salud pública
}

\author{
Editorial: Dossier Oral Microbiology and Public Health
}

Editorial: Dossier microbiologia oral e saúde pública

Fredy OMAR GAMbOA JAIMES

Pontificia Universidad Javeriana, Bogotá, Colombia. gamboa@javeriana.edu.co; https://orcid.org/0000-0003-2847-9837

\section{Dabeiba Adriana García Robayo}

Pontificia Universidad Javeriana, Bogotá, Colombia. garciad@javeriana.edu.co; https://orcid.org/0000-0003-0770-9138

Correspondencia: gamboa@javeriana.edu.co; garciad@javeriana.edu.co

doi: https://doi.org/10.11144/Javeriana.uo39.edmo

Cómo citar: Gamboa Jaimes FO, García Robayo DA. Editorial: Dossier microbiología oral y salud pública. Univ Odontol. 2020; 39. https://doi.org/10.11144/Javeriana.uo39.edmo 


\section{RESUMEN}

Tenemos el gusto de presentar a nuestros lectores el dossier temático Microbiología oral y salud pública. Este dossier es producto de la interacción multidisciplinar de profesores investigadores de la Facultad de Odontología y del Centro de Investigaciones Odontológicas (CIO) de la Pontificia Universidad Javeriana (PUJ) de Bogotá y refleja de manera clara, destacada y pertinente la producción académica que han tenido en los últimos años. El CIO pertenece a la Facultad de Odontología de la PUJ. Está comprometido con la generación de conocimiento en las áreas de ciencias básicas (microbiología, inmunología, genética, bioquímica y física), clínicas y sociales relacionadas con enfermedades orales (infecciosas y no infecciosas) y sistémicas, que contribuyan a solucionar los problemas de salud pública de la población colombiana.

\section{Palabras clave}

biopelícula; carcinoma escamocelular oral; caries dental; dossiers temáticos; ecosistema oral; educación preescolar; enfermedad periodontal; higiene oral; microbiología oral; microbiota oral; odontología; P. gingivalis; S. mutans; salud publica oral

\section{ABSTRACT}

We are pleased to present to our readers the thematic dossier Oral Microbiology and Public Health. This dossier is the product of the multidisciplinary interaction of research professors from the Faculty of Dentistry and the Dental Research Center (CIO) of the Pontificia Universidad Javeriana (PUJ) of Bogotá and reflects in a clear, prominent and pertinent way the academic production that 
they have had in recent years. The CIO belongs to the PUJ School of Dentistry. It is committed to the generation of knowledge in the areas of basic sciences (microbiology, immunology, genetics, biochemistry and physics), clinical and social related to oral diseases (infectious and noninfectious) and systemic, which contribute to solving public health problems of the Colombian population.

\section{Keywords}

biofilm; dental caries; dentistry; oral ecosystem; oral hygiene; oral microbiology; oral microbiota; oral public health; oral squamous cell carcinoma; $P$. gingivalis; periodontal disease; preschool education; S. mutans; thematic dossiers

\section{RESUMO}

Temos o prazer de apresentar aos nossos leitores o dossiê temático Microbiologia Oral e Saúde Pública. Este dossiê é fruto da interação multidisciplinar de docentes pesquisadores da Faculdade de Odontologia e do Centro de Investigaciones Odontológicas (CIO) da Pontifícia Universidade Javeriana (PUJ) de Bogotá e reflete de forma clara, destacada e pertinente a produção acadêmica que realizaram nos últimos anos. O CIO pertence à Faculdade de Odontologia do PUJ. Está empenhada na geração de conhecimentos nas áreas das ciências básicas (microbiologia, imunologia, genética, bioquímica e física), clínicas e sociais relacionadas com as doenças orais (infecciosas e não infecciosas) e sistémicas, que contribuam para a resolução de problemas de saúde pública da população colombiana. 


\section{Palavras-chave}

biofilme; carcinoma de células escamosas oral; cárie; dossiês temáticos; ecossistema oral; educação pré escolar; doença periodontal; higiene oral; microbiologia oral; microbiota oral; odontologia; P. gingivalis; S. mutans; saúde pública oral

Tenemos el gusto de presentar a nuestros lectores el dossier temático Microbiología oral y salud pública. Este dossier es producto de la interacción multidisciplinar de profesores investigadores de la Facultad de Odontología y del Centro de Investigaciones Odontológicas (CIO) de la Pontificia Universidad Javeriana (PUJ) de Bogotá y refleja de manera clara, destacada y pertinente la producción académica que han tenido en los últimos años. El CIO es un centro de investigación dependiente de la Facultad de Odontología de la PUJ, comprometido con la generación de conocimientos en las áreas de ciencias básicas (microbiología, inmunología, genética, bioquímica y física), clínicas y sociales relacionadas con enfermedades orales (infecciosas y no infecciosas) y sistémicas, que contribuyan a solucionar los problemas de salud pública de la población colombiana.

La cavidad bucal es uno de los nichos ecológicos del cuerpo humano con mayor diversidad en microbiota. El estado de equilibrio o eubiosis de la microbiota oral es de vital importancia en el desarrollo de las funciones de los diferentes ecosistemas orales, ya que entre otras acciones lleva a una adecuada respuesta inmune frente a la invasión o multiplicación de los patógenos que conducen a enfermedades. En términos ecológicos, muchas enfermedades de cavidad oral son consecuencia de un desequilibrio en el ambiente oral que lleva al predominio de una flora en concreto. Entre estas enfermedades encontramos la caries dental, la enfermedad periodontal y más 
recientemente el cáncer oral y de orofarínge. Las dos primeras patologías presentan una elevada incidencia y prevalencia en la población colombiana pediátrica y adulta, respectivamente, que las convierte en enfermedades de importancia en salud pública oral. Por su parte, el cáncer oral y orofaríngeo presenta en Colombia y en el mundo un aumento considerable en su incidencia en las últimas décadas, principalmente en personas menores de 40 años, situación que enciende las alarmas para diseñar de manera urgente adecuadas estrategias de prevención. En los últimos años las técnicas de biología molecular han permitido ampliar los conocimientos en microbiología oral al reconocer muchos géneros y especies que aún no han podido ser cultivados y caracterizados al día de hoy. También es necesario señalar la importancia de la implementación y puesta a punto de procesos educativos en salud oral en espacios escolares, como estrategia fundamental de la promoción de la salud, por ser considerada una herramienta efectiva para el desarrollo de prácticas protectoras en salud oral.

En este sentido los autores del dossier quieren compartir algunos de los estudios y experiencias de investigación llevadas a cabo durante más de 20 años en la temática de microbiología oral y sus implicaciones en salud pública. El presente dossier cuenta con la selección de 6 artículos que incluye: 1. Una revisión sistemática de la literatura, para identificar la composición bacteriana presente en población de 3 a 12 años y su relación con el estado gingival y periodontal, y planteada por la poca información existente en esta población en comparación con población adulta; 2. El segundo artículo describe y compara las características morfológicas, microbiológicas e inmunológicas del periodonto en población pediátrica y adulta, con el fin de entender las diferencias en las manifestaciones clínicas de la enfermedad periodontal que se incrementan con la edad; 3. El tercer artículo presenta experiencias desde un diagnóstico histopatológico en 
displasia epitelial y cáncer escamocelular oral y orofaringeo, así como entender el papel del Virus del Papiloma Humano (VPH) en esta patología. En este mismo artículo, también se presentan los hallazgos en la presencia de algunas bacterias orales asociadas a este tipo de cáncer en pacientes con y sin carcinoma de orofarínge y descripción de la co-infeccion Streptococcus anginosus- VPH relacionada con el pronóstico del paciente; 4. En el artículo "Determinantes sociales de la salud, microbioma oral y sus implicaciones prácticas en ámbitos escolares” se discute la implementación de estrategias en salud oral como soporte de programas de promoción de la salud, para lo cual es importante establecer una alianza entre la academia y las instituciones educativas a través de los profesionales de la salud y la comunidad escolar en general, teniendo en cuenta los determinantes sociales de la salud; 5. En el quinto artículo "Caries dental: de la placa ecológica a las decisiones clínicas" se presenta la evolución de la definición de la caries dental a partir del papel de las bacterias y su impacto en las decisiones clínicas, las acciones para abarcar la definición actual de caries dental y las acciones ajustadas al modelo ICCMS (International Caries Classification and Management System) para llevarlo a la práctica; 6. Finalmente en el sexto artículo "Características microbiológicas y moleculares de microorganismos de importancia en caries dental y enfermedad periodontal: aportes de investigación en Colombia" se describen las estrategias de aislamiento e identificación, susceptibilidad antimicrobiana, biotipos, fenotipos y características genotípicas de S. mutans y $P$. gingivalis, microorganismos, respectivamente, de gran importancia en caries dental y enfermedad periodontal.

En conclusión, se espera que el dossier presentado contribuya de manera positiva a entender y conocer las características fenotípicas y genotípicas de microorganismos implicados en enfermedades de cavidad oral y que unido este conocimiento profundo a la epidemiología clásica 
pueda ayudar a la construcción, formulación, implementación y evaluación de mejores políticas en salud publica oral.

Fredy Omar Gamboa Jaimes Dabeiba Adriana García Robayo

Editores invitados 